\title{
ESTIMATION AND CORRECTION OF SCINTILLATION EFFECTS ON SPACEBORNE P-BAND SAR IMAGES
}

\author{
Jun Su Kim ${ }^{\text {I) }}$, Konstantinos P. Papathanassiou ${ }^{1)}$, Shaun Quegan ${ }^{2)}$ and Neil Rogers ${ }^{2)}$ \\ 1) German Aerospace Center (DLR) \\ Microwaves and Radar Institute (DLR-HR) \\ P.O.Box 1116, D-82234 Weßling, Germany \\ Tel/Fax: ++49-8153-28-2343/1449, Email: Junsu.Kim@,dlr.de \\ 2) Centre for Terrestrial Carbon Dynamics, University of Sheffield
}

\begin{abstract}
A correction method for distortions induced by ionospheric scintillation effects on P-band quad-pol synthetic aperture radar (SAR) data acquired in polar and high latitude regions is presented. For this the estimation of the Faraday rotation (FR) is converted to ionospheric phase and used for the correction of the scintillation. The correction is performed on partially focused SLC data in order to compensate the ionosphere-induced phase and the distortion of the timeDoppler history. The degree of defocusing depends on the altitude of the ionosphere. The performance of the new correction method is tested on P-band simulated data for various ionospheric scenarios.
\end{abstract}

Index Terms - ionosphere, polarimetry, focusing

\section{INTRODUCTION}

Microwave pulses propagating through the ionosphere change their phase and group velocities. This can cause measurable distortions on the focused SAR images [1], [2], [3]. In particular, small scale irregularities in the ionosphere distort the time-Doppler history of individual scatterers, so that the focused image loses its contrast and its correct phase information [1], [2]. Due to the small length and time scales of the ionospheric irregularities, no external/ independent observations are available to be used in correction. In this paper we propose a new adaptive correction method based on polarimetric SAR data using the anisotropic characteristics of the ionosphere. The proposed methodology is validated using simulated P-band spaceborne SAR data in accordance with the characteristics of BIOMASS, a candidate Earth Explorer mission of the European Space Agency (ESA).

\section{THEORETICAL BACKGROUND}

The ionospheric phase distortion and Faraday Rotation (FR) are both proportional to the total electron content (TEC) [2], [3], [4], which characterises the state of the ionosphere. The one-way FR, $\Omega$, is linked to TEC as

$$
\Omega=\zeta \frac{e \vec{B} \cdot \hat{\kappa}}{c m f_{0}^{2}} T E C,
$$

where $e$ and $m$ are the charge and mass of an electron, respectively, $\vec{B}$ is the Earth's magnetic field, $\hat{\kappa}$ is the unit propagation vector of the SAR's microwave pulse, $c$ is the speed of light, $f_{0}$ is the centre frequency, and $\zeta$ is a constant which is defined as

$$
\zeta=\frac{e^{2}}{8 \pi^{2} \varepsilon_{0} m}=40.31 \mathrm{~m}^{3} / \mathrm{s}^{2} .
$$

The Earth's magnetic field is a function of longitude, latitude and altitude. The value of $\vec{B} \cdot \hat{\kappa}$ varies in the range $\pm 50,000 \mathrm{nT}$ interval at the typical ionospheric altitude of $350 \mathrm{~km} \mathrm{[5]} \mathrm{with} \mathrm{respect} \mathrm{to} \mathrm{the} \mathrm{general} \mathrm{sun-synchronous}$ orbit associated with $30^{\circ}$ incidence angle. The (two-way) phase advance of the signal that travels through the ionosphere characterised by a given TEC is

$$
\phi=\frac{4 \pi \zeta}{c f_{0}} T E C \text {. }
$$

Combining Eq. 1 and Eq. 3 one gets the relation between the FR and the ionospheric phase

$$
\phi=\frac{4 \pi m f_{0}}{e \vec{B} \cdot \hat{\kappa}} \Omega .
$$

The constant of the proportionality is 777 at P-band (435 $\mathrm{MHz})$ at high latitudes and 2269 at L-band $(1.27 \mathrm{GHz})$, taking $\vec{B} \cdot \hat{\kappa}=40,000 \mathrm{nT}$ in both cases. These figures increase as the latitude decreases. At low frequencies Eq. 4 can be used to compensate scintillation-induced phase screens from estimates of $\Omega$.

\section{DATASETS}

In the absence of real spaceborne SAR P-band data, simulation of spaceborne data using airborne data is a pragmatic approach. Airborne data acquired during the BIOSAR-I campaign by DLR's E-SAR system over the Remningstorp test site in Sweden in May 2007 was applied in this study. In order to obtain the simulated spaceborne BIOMASS data products: i) the spatial resolution was degraded in range $(50 \mathrm{~m}$ on the ground for $6 \mathrm{MHz})$ and 


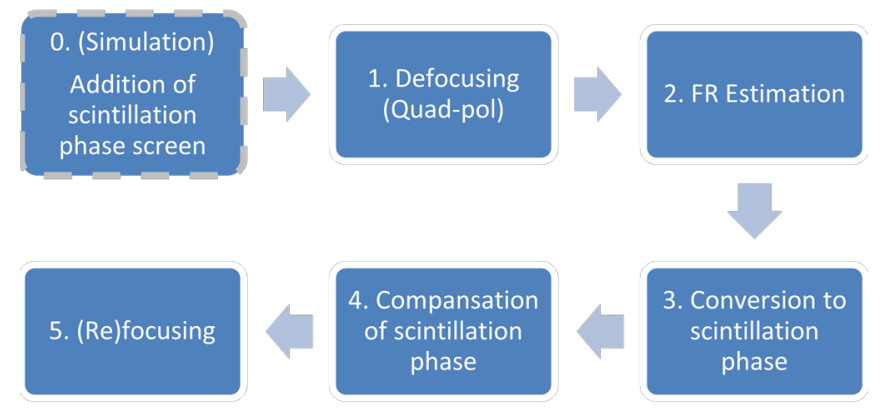

Figure 1: Block diagram of proposed correction method.

azimuth $(12.5 \mathrm{~m})$; ii) the noise level was increased; and iii) the range and azimuth ambiguity levels were increased.

The ionospheric phase screens were calculated by the University of Sheffield based on a power-law irregularity spectrum derived from the WBMOD ionospheric model from North West Research Associates Inc. [6]. The WBMOD model generates ionospheric realisations with a TEC variance corresponding to a set of parameters that depend on geographic location, orbit, ionospheric anisotropy, power-law value, etc. A sun-synchronous duskdawn orbit at $580 \mathrm{~km}$ altitude, inclination of $98^{\circ}$ and an offnadir angle of $30^{\circ}$ were applied. Six ionospheric scintillation scenarios were realised, given by the permutation of three different planetary indices, $\mathrm{Kp} 1,3$ and 7, and two percentiles of occurrence of the $\mathrm{C}_{\mathrm{k}} \mathrm{L}$ (integrated strength of turbulence) Index of $50 \%$ and $90 \%$. In order to concentrate on the effects of scintillations, the mean background ionosphere was removed.

\section{CORRECTION STEPS}

Figure 1 shows the processing steps in the proposed scintillation correction method. The first step is the simulation of a distorted image using the P-band datasets and ionospheric phase screens discussed in Section 3, which of course is not required in the actual correction scenario. The process of focusing and defocusing at the height of the ionospheric layer is explained in Section 4.1. Section 5 is dedicated to the FR estimation step. The conversion between FR and scintillation phase is given by Eq. 4 .

\subsection{Addition of Scintillation}

The addition of the scintillation phase change is carried out at the ionospheric altitude. As the SLC images are focused at the ground level, they have to be defocused to the ionospheric altitude before adding the scintillation phase screen. In addition, to complete the addition of the scintillation phase, the SLC image must again be focused at ground level.

The time-Doppler history is a function of the nearest approach to a scatterer. In order to focus the scene at the
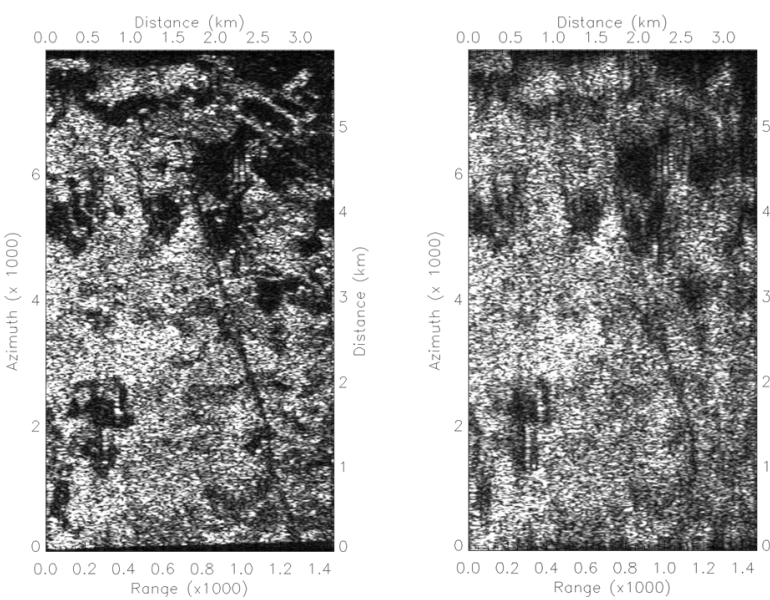

Figure 2: Simulated P-band image before scintillation (left) and after scintillation of $\mathrm{Kp}=7$ and $\mathrm{C}_{\mathrm{k}} \mathrm{L} \mathrm{CI}=90 \%$ (right)

ionospheric altitude, the matched filter should be adapted to the ionospheric range distance. Such a focusing can be achieved by modifying the azimuth frequency vector $f_{a}$ of [7, p. 31], with respect to the ionospheric altitude:

$$
\phi_{\text {decompr }}\left(f_{a} ; r_{\text {iono }}\right)=-\frac{4 \pi}{\lambda} r_{\text {iono }}\left(\sqrt{1-\left(\frac{f_{a} \lambda}{2 v}\right)^{2}}-1\right) .
$$

Here $r_{i o n o}$ is the range to the assumed ionospheric altitude and $\phi_{\text {decompr }}$ is a phase multiplied in the frequency domain of the SLC data. Note that the multiplication of Eq. 5 is carried out in the frequency domain. Its time domain equivalent is a partially focused (equivalently, focused at the ionospheric altitude) SLC. Now the scintillation phase $(\phi)$ is multiplied to simulate the contaminating phase:

$$
S L C^{\prime}=S L C \cdot e^{i \phi} .
$$

The subsequent multiplication of $-\phi_{\text {decompr }}$ in the frequency domain of $S L C^{\prime}$ provides the ground-level focused SLC, disturbed by the scintillation phase.

Figure 2 compares the simulated P-band SLC data before and after the scintillation distortion. After distortion, the scene features are smeared along azimuth and the contrast is decreased. With increasing $\mathrm{Kp}$ and $\mathrm{C}_{\mathrm{k}} \mathrm{L}$ levels, ionospheric scintillation increases.

\section{FR SIMULATION}

The most reliable FR estimator is that proposed by Bickel \& Bates [8], [9]. The variance of the FR estimates, $\sigma_{\Omega}^{2}$, associated with a given SNR level by [10]

$$
\sigma_{\Omega}^{2}=\frac{1}{16}\left(\begin{array}{c}
\frac{\pi^{2}}{3}-\pi \sin ^{-1} \gamma_{\mathrm{SNR}} \\
+\left(\sin ^{-1} \gamma_{\mathrm{SNR}}\right)^{2}-\frac{\mathrm{Li}_{2} \gamma_{\mathrm{SNR}}^{2}}{2}
\end{array}\right) .
$$




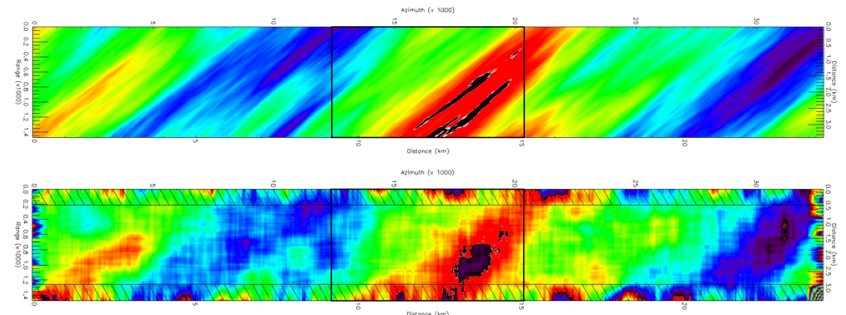

Figure 3: Original ionospheric phase screen (top) and after noise addition and spatial averaging (bottom). $\mathrm{Kp}=1$, and $\mathrm{C}_{\mathrm{k}} \mathrm{L} \mathrm{CI}=90 \%$ is applied.

where $\gamma_{\mathrm{SNR}}$ is given by

$$
\gamma_{\mathrm{SNR}}=\frac{S N R}{1+S N R},
$$

and $\mathrm{Li}_{2}$ is the Euler dilogarithm.

A noise component with Gaussian distribution and a given standard deviation is added to the FR inferred from the scintillation phase screens. After the noise addition, the obtained FR map is smoothed to reduce the noise contribution and then converted back to an ionospheric scintillation phase.

Figure 3 shows examples of the applied/estimated scintillation phase screens. At the top, the scintillation phase screen for $\mathrm{Kp}=1$ and $\mathrm{C}_{\mathrm{k}} \mathrm{L}$ CI $90 \%$ conditions used to distort the original P-band SLC data is shown. Below, the recovered scintillation phase screen that is used for correcting the distorted SLC image is shown. The noise in the FR estimation has degraded the high frequency component of the scintillation phase screen.

\section{RESULTS}

The distorted SLCs are corrected using the scintillation phase screen which is derived from the FR estimations of Section 5. The performance of the correction is evaluated in terms of the correlation coefficient between the corrected and the original (undistorted) SLC data (see Figure 4). The best correlation coefficients are expected at high latitudes in strong backscattering areas, and for rather quiet scintillation scenarios (i.e. low $\mathrm{C}_{k} \mathrm{~L}$ and low $\mathrm{Kp}$ ). The correction performance with respect to an increasing estimation window, or the number of looks, shows the positive effect of noise smoothing at the cost of resolution loss. The correlation coefficient reaches its maximum for a window size of $4 \mathrm{~km}^{2}$, in the appropriate range of SNR and geomagnetic longitudes.

The correlation coefficient between the original and the corrected SLC data for various parameters has been estimated. As well as the ionospheric scintillation scenarios, the estimation window size $\left(1 \mathrm{~km}^{2}, 2.25 \mathrm{~km}^{2}\right.$ and $\left.4 \mathrm{~km}^{2}\right)$, the radar backscatter $\left(-10 \mathrm{~dB}\right.$ and $-15 \mathrm{~dB}$; assumed $\sigma_{\mathrm{NESZ}}$ is -28 $\mathrm{dB}$ for BIOMASS), and geomagnetic latitude ( $\vec{B} \cdot \hat{\kappa}=40,000$ and 30,000 nT) have been varied. In addition the effect of the ionospheric altitude estimation error is also evaluated.
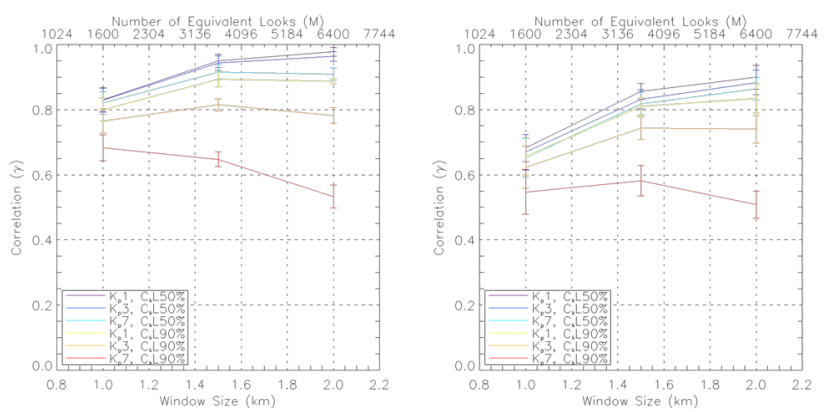

a) $\sigma_{0}=-10 \mathrm{~dB}, \mathrm{~B}=40,000 \mathrm{nT}$

b) $\sigma_{0}=-15 \mathrm{~dB}, \mathrm{~B}=40,000 \mathrm{nT}$
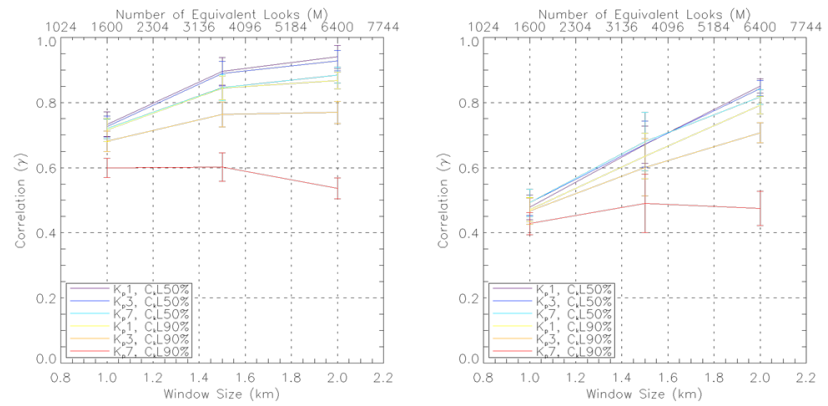

c) $\sigma_{0}=-10 \mathrm{~dB}, \mathrm{~B}=30,000 \mathrm{nT}$

d) $\sigma_{0}=-15 \mathrm{~dB}, \mathrm{~B}=30,000 \mathrm{nT}$

Figure 4: Correlation coefficients for different combinations of parameters

For each set of parameters, 10 random noise realisations for the FR estimation have been carried out.

\subsection{Noise controlling parameters}

The success of the scintillation correction is directly dependent on the quality of the reconstruction of the scintillation phase screen. The associated parameters are: i) the scintillation scenarios, ii) the size of the FR estimation window, iii) the SNR level, and iv) the geomagnetic latitude. Figure 4 shows the change of the correlation coefficients after correction, with their estimated standard deviations. The FR estimation window size is along the abscissa and the estimated correlation coefficients and their standard deviations are drawn along the ordinates. The six scintillation scenarios are distinguished with lines of different colours: quiet ionospheric conditions $\left(\mathrm{C}_{\mathrm{k}} \mathrm{L}\right.$ $\mathrm{CI}=50 \%$ ) have cold colours while warm colours indicate active conditions $\left(\mathrm{C}_{\mathrm{k}} \mathrm{L} \quad \mathrm{CI}=90 \%\right)$. For the different geomagnetic latitudes and the backscattering levels, four plots are presented.

We can observe that i) the use of a larger number of looks improves correction performance; ii) this advantage reduces when the correction conditions are more severe; and iii) for the more disturbed scintillation scenarios, the number of looks for optimum correlation reduces.

Lower SNR levels increase the variance of the FR estimation. The quality of the scintillation phase screen 


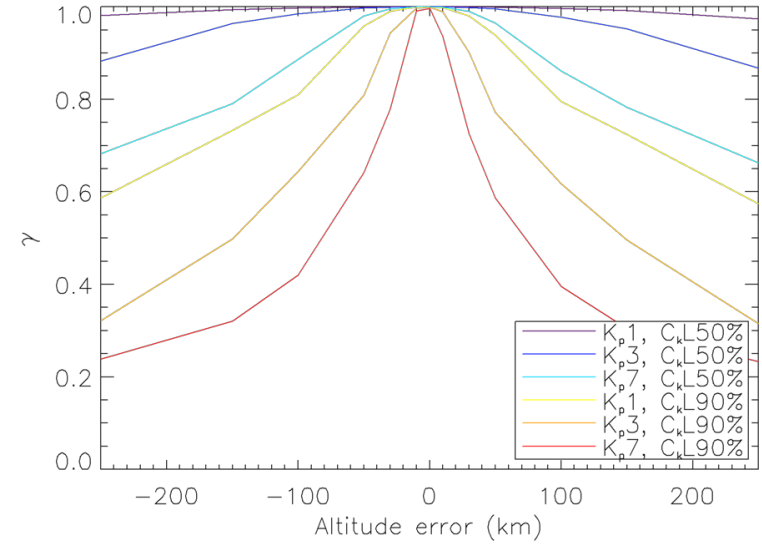

Figure 5: Change of correlation coefficient as a function of the error in ionospheric altitude estimation.

estimation degrades, and the correction performance is limited.

The same process happens at lower geomagnetic latitudes. During the conversion of FR to a scintillation phase screen using Eq. 4, the variance of the FR estimate is amplified because of the reduction of the $\vec{B} \cdot \hat{\kappa}$ term. This adverse effect degrades the quality of the inferred scintillation phase screen, and eventually the correction performance.

\subsection{Altitude of the ionosphere}

In the simulated data, the ionospheric screen has been introduced at an altitude of $350 \mathrm{~km}$. In a realistic scenario, however, the ionospheric altitude is unknown and has to be estimated or assumed. Either may be affected by errors. The effect of the ionospheric altitude error is simulated by moving the induced ionospheric phase screen to an altitude different from that at which it was induced. The results are shown in Figure 5. Here we applied the original ionospheric phase screens (top of Figure 3) but controlled the value of $r_{\text {iono }}$ in Eq. 5 in the compensation step.

For larger altitude errors, positive or negative, decreasing correlation trends are observed. The amount of decorrelation strongly depends on the ionospheric scenario. For stronger ionospheric scintillation, the decorrelation increases rapidly, indicating the need for more accurate knowledge of the ionospheric altitude. The decreasing trend is almost symmetric for positive and negative errors.

\section{CONCLUSION}

A new methodology for scintillation correction has been tested using simulated P-band data for the mission parameters of the proposed BIOMASS mission. Its long wavelength facilitates the conversion of FR to a scintillation phase screen, but its narrow bandwidth (or relatively coarse resolution) limits the quality of the reconstructed scintillation phase screen.

We propose a correlation coefficient of 0.9 as a good benchmark to judge the quality of the correction. The observations indicate that: i) the correlation threshold of 0.9 is relatively easy to attain when the radar backscatter is -10 $\mathrm{dB}$, and is just met when the radar backscatter is $-15 \mathrm{~dB}$ and the number of looks exceeds $6,400\left(4 \mathrm{~km}^{2}\right.$ window) for $\mathrm{C}_{\mathrm{k}} \mathrm{L}$ $\mathrm{CI}=50 \%$; ii) the geomagnetic latitude is important, but for mid- to high-latitudes (B $>30,000 \mathrm{nT}$ ), it is not a critical factor for $\mathrm{C}_{\mathrm{k}} \mathrm{L} \mathrm{CI}=50 \%$; and iii) an altitude estimation error of $\pm 50 \mathrm{~km}$ is acceptable for the condition $\mathrm{C}_{\mathrm{k}} \mathrm{L} \mathrm{CI}=50 \%$.

\section{REFERENCES}

[1] Z.-W. Xu, J. Wu and Z.-S. Wu, "A Survey of Ionospheric Effects on Spacebased Radar," Waves in Random Media, vol. 14, pp. 189-273, 2004.

[2] D. P. Belcher, "Theoretical Limits on SAR imposed by the ionosphere," IET Radar Sonar Navig., vol. 2, no. 6, pp. 435-448, 2008.

[3] F. Meyer, R. Bamler, N. Jakowski and T. Fritz, "The potential of Low-Frequency SAR Systems for Mapping Ionospheric TEC Distributions," IEEE Geoscience and Remote Sensing Letters, vol. 3, no. 4, pp. 560-564, October 2006.

[4] J. S. Kim, A. Dankelmeyer and K. Papathanassiou, "Correction of ionospheric distortions in low frequency interferometric SAR data," in IGARSS2011, Vancouver, Canada, 2011.

[5] P. A. Wright, S. Quegan, N. S. Wheadon and C. D. Hall, "Faraday rotation Effects on L-Band Spaceborne SAR Data," IEEE Transactions on Geoscience and Remote Sensing, vol. 41, no. 12, pp. 2735-2744, December 2003.

[6] J. A. Secan, "WBMOD Ionospheric Scintillation Model - An abbreviated user's guide (Version 15)," Northwest Resarch Associates, Inc., Tucson, AZ, 2007.

[7] P. I. Prats, Airborne Differential SAR Interferometry, Ph.D. Disseration, Universitat Politécnica de Catalunya, 2005.

[8] S. H. Bickel and R. H. T. Bates, "Effects of MagnetoIonic Propagation on the Polarization Scattering Matrix," Proc. IRE, vol. 53, pp. 1089-1091, 1965.

[9] J. S. Kim and K. Papathanassiou, "Faraday rotation estimator performance analysis," in Proceedings of EUSAR 2010, Aachen, Germany, 2010.

[10] R. F. Hanssen, Radar Interferometry, Dordrecht, The Netherlands: Kluwer Academic Publishers, 2001. 